\title{
A Novel Algorithm for Ship Detection in SAR Imagery Based on the Wavelet Transform
}

\author{
Mariví Tello, Carlos López-Martínez, Member, IEEE, and Jordi J. Mallorqui, Member, IEEE
}

\begin{abstract}
Carrying out an effective control of fishing activities is essential to guarantee a sustainable exploitation of sea resources. Nevertheless, as the regulated areas are extended, they are difficult and time consuming to monitor by means of traditional reconnaissance methods such as planes and patrol vessels. On the contrary, satellite-based synthetic aperture radar (SAR) provides a powerful surveillance capability allowing the observation of broad expanses, independently from weather effects and from the day and night cycle. Unfortunately, the automatic interpretation of SAR images is often complicated, even though undetected targets are sometimes visible by eye. Attending to these particular circumstances, a novel approach for ship detection is proposed based on the analysis of SAR images by means of the discrete wavelet transform. The exposed method takes advantage of the difference of statistical behavior among the ships and the surrounding sea, interpreting the information through the wavelet coefficients in order to provide a more reliable detection. The analysis of the detection performance over both simulated and real images confirms the robustness of the proposed algorithm.
\end{abstract}

Index Terms-Multiresolution analysis, ship detection, synthetic aperture radar (SAR) imagery, wavelet transform.

\section{INTRODUCTION}

A LARGE amount of investigations have proven that satellite-based synthetic aperture radar (SAR) can be used for ship detection purposes [1]. Particularly, in Europe, the Improving Fisheries Monitoring by Integrating Passive and Active Satellite Technologies Project (IMPAST) aims at developing a preoperational system for the control of fishing activities which combines information from satellite imagery and from the Vessel Monitoring System (VMS), while the Detection and Classification of Marine Traffic from Space Project (DECLIMS) compares the performance of existing ship detection and classification tools based on spaceborne systems [2]. One observation extracted during the IMPAST campaigns has been that automatic detection success is proved lower than expected.

Since the dimensions and the shape of the signature of the target are, a priori, unknown and very diverse, conventional ship detection algorithms are conceived to discriminate an exceptionally bright localized pattern, according to an established decision rule. This reasoning presents two drawbacks. On the

Manuscript received October 18, 2004; revised January 14, 2005. This work was supported in part by the Spanish MCYT and FEDER funds under Project TIC2002-04451-C02-01 and in part by the European Commission in the scope of IMPAST Project Q5RS-2001-02266.

M. Tello and J. J. Mallorqui are with the Universitat Politècnica de Catalunya, Barcelona, Spain (e-mail: marivi.tello@ tsc.upc.es; mallorqui@tsc.upc.es).

C. López-Martínez is with the Université de Rennes 1, Institut d'Electronique et de Télécommunications de Rennes, Centre National de la Recherche Scientifique (CNRS UMR 6164), Rennes, France.

Digital Object Identifier 10.1109/LGRS.2005.845033 one hand, the adjustment of thresholds involved in the decision step is complicated and it relies on the existence of a considerable contrast between the vessels to be detected and the sea clutter, which is not always reached, e.g., for wooden boats. On the other hand, oceanic SAR imagery is affected by different kinds of disturbances, such as speckle or marine discontinuity effects due to random changes in bathymetry and wind currents. All these irregular structures are suspect of being perceived as potential positive detections - generating false alarms - or they can yield to the failure of the system, hiding some targets. These remarks imply that it would be more effective facing the detection not only taking exclusively into account the intensity characteristics of the image but also studying its very localized statistical behavior.

Another interesting observation is that, surprisingly, undetected vessels are sometimes visible by eye. The eye sees a texture over two fundamental properties: the orientation of its different elements and its frequency content, simultaneously performing over the observed scene a selective filtering in frequency and in orientation. Moreover, the human visual system perceives images in a multiscale way, as it is able to focus on different elements at different scales.

Thus, the proposed method plans on conveniently reconciling the previous aspects by means of the wavelet theory. As the wavelet transform (WT) provides a multiscale analysis of the images, according to their spatial and frequency characteristics, multiresolution processing with wavelets is a suitable tool for modeling the operation of the human vision. Furthermore, wavelet theory permits characterizing the statistical behavior of a function locally.

After presenting some useful tools provided by the wavelet theory, the novel approach for ship detection in SAR imagery will be presented, theoretically justified and tested over simulated and real data.

\section{WAVELET TOOLS}

In this section, some of the aspects of the WT will be briefly discussed. For a more extensive mathematical study, [3] must be consulted.

\section{A. Multiresolution Analysis With the Wavelet Transform}

The WT proposes the study of a complex phenomenon, dividing it into different simpler pieces. Mathematically, this implies projecting it in a function space, in which it is located by measuring its degree of similarity with each basic function or atom, i.e.,

$$
W f=\left[n, a^{j}\right]=\sum_{m=0}^{N-1} f[m] \psi_{j}^{*}[m-n]
$$


where $f$ is the discrete signal, $\psi_{j}$ is a discrete wavelet atom and $a^{j}$ is the scale.

In a WT, the basic functions come from dilations and translations of a "mother wavelet" $\psi$ localized in both time and frequency

$$
\psi_{j}[n]=\frac{1}{\sqrt{a^{j}}} \psi\left(\frac{n}{a^{j}}\right) .
$$

Each term of the basis, therefore, allows the representation of the signal at a particular frequency scale and time/space position. Consequently, WT can focus on structures with a "zooming" procedure. In order to obtain a complete representation, a scaling function $\phi$, which is an aggregation of wavelets at scales larger than 1, is introduced. The modulus of its Fourier transform is defined by [3]

$$
|\hat{\phi}(\omega)|^{2}=\int_{\omega}^{+\infty} \frac{|\hat{\psi}(\xi)|^{2}}{\xi} d \xi
$$

and the complex phase of $\hat{\phi}(\omega)$ can be arbitrarily chosen.

For two-dimensional (2-D) signals, a wavelet orthonormal basis is constructed with separable products of a scaling and a wavelet function which can be assimilated to low-pass (L) and bandpass $(\mathrm{H})$ filters, respectively

$$
\begin{aligned}
l[n] & =\frac{1}{\sqrt{2}}\left\langle\phi\left(\frac{t}{2}\right), \phi(t-n)\right\rangle \\
h[n] & =\frac{1}{\sqrt{2}}\left\langle\psi\left(\frac{t}{2}\right), \phi(t-n)\right\rangle .
\end{aligned}
$$

Three wavelets are then defined (HL, LH, HH), each of them extracting image details for a given orientation, while LL is a low-pass filtered version of the original image. More specifically, the WT performs at each iteration a separation of the different frequency components of the original image, according to their orientation: coefficients of large amplitude in the different detail subbands correspond to horizontal, vertical, and diagonal edges (Figs. 1 and 2).

\section{B. Singularity Detection}

It is well known that the WT of a function at a particular point depends on the values of the function smoothed by the wavelet in a neighborhood, whose dimensions are proportional to the scale. Thus, for fine scales, the WT shall provide localized information about the variation of the function around a certain point or, namely, about its local regularity. Therefore, irregularities will be sharpened in the transformed domain or, more specifically, the existence of discontinuities in the original signal will result in local maxima in the WT.

\section{NOVEl APPROACH FOR SHIP DETECTION IN SAR IMAGERY}

The objective of the proposed algorithm is to detect the presence and the position of a ship in a SAR image (Fig. 1), which means localizing an irregular structure in a noisy background which presents itself different types of discontinuities at different scales. As the WT, by means of the multiresolution analysis, allows to adapt the resolution to the extraction of the irreg-

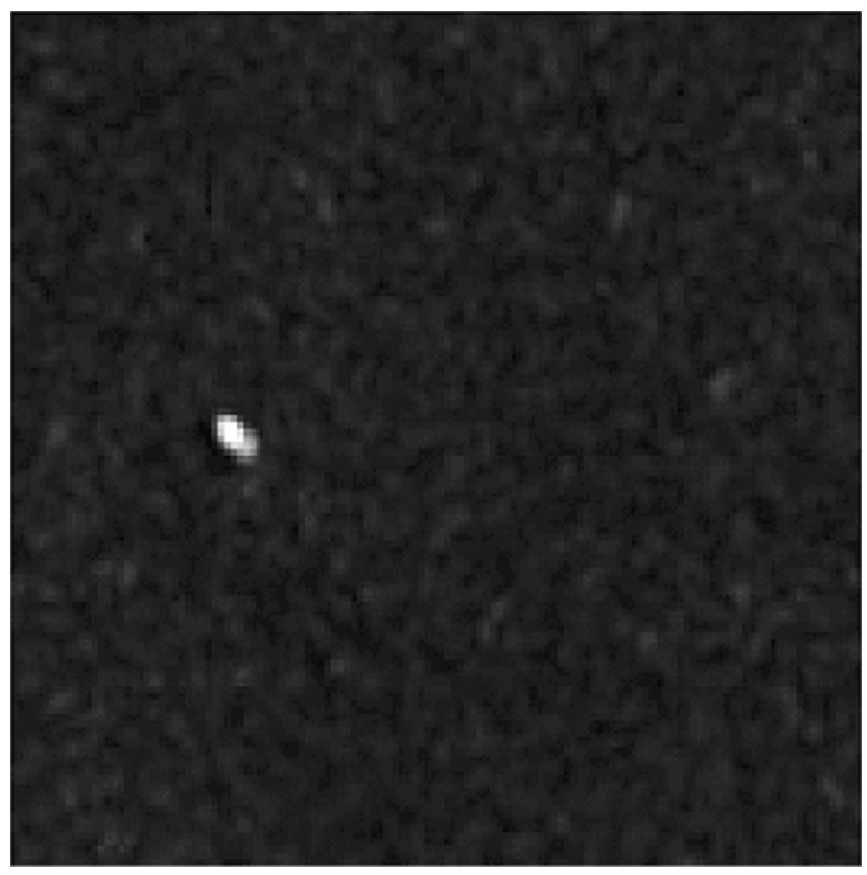

Fig. 1. Fragment of real Radarsat image $(256 \times 256$ pixels $)$ presenting an easily detectable ship.
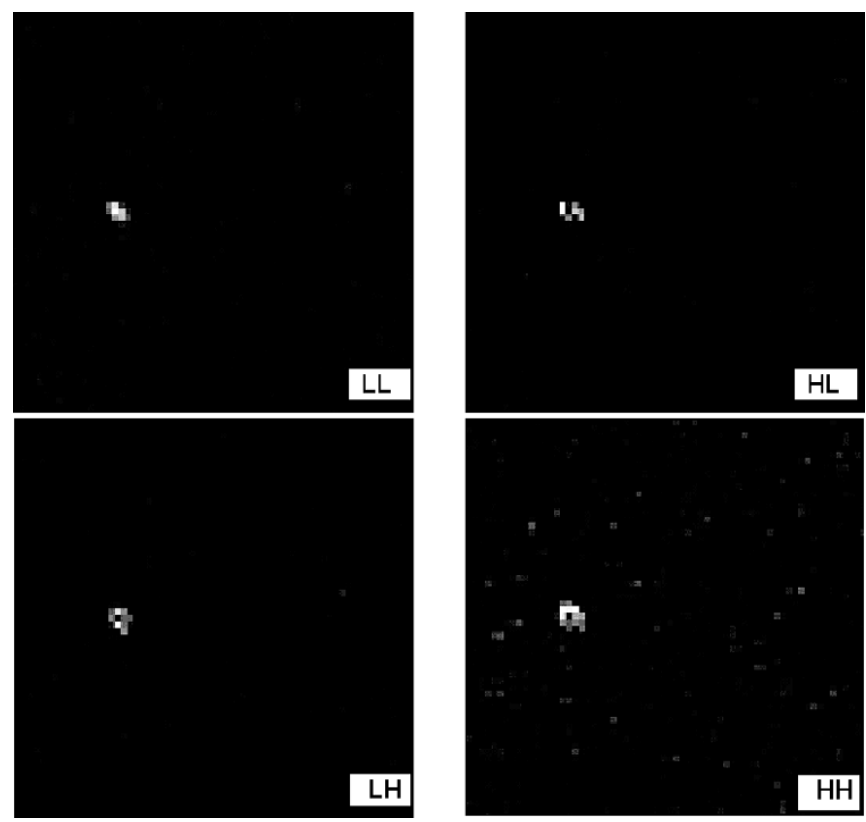

Fig. 2. Result of the application of the DWT by means of the dyadic pyramidal algorithm with Haar coefficients. Each detail subband enhances edges in a particular direction (HL: vertical edges; LH: horizontal edges; HH: diagonal edges).

ularities of the signal—noticeable by maxima in the WT modulus - this tool seems to be very well suited for analyzing these multiscale discontinuities.

The primary features of the WT (locality, multiresolution and edge detection) usually assume the decorrelation of the detail subbands. However, it has been shown that the WT is not able to remove the most local dependencies, due to regular or homogeneous spatial structures and patterns, as it can be seen in Fig. 2. 


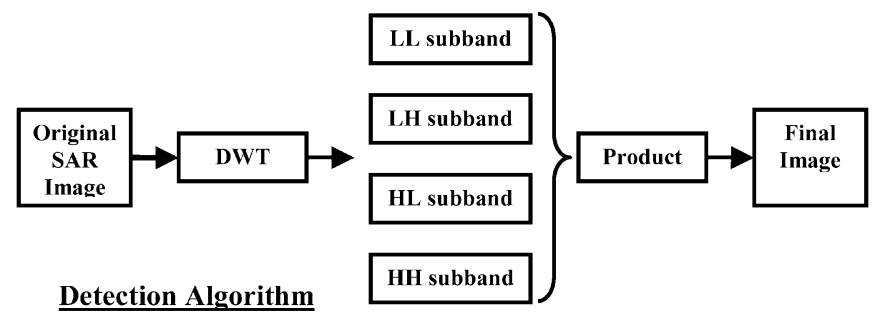

Fig. 3. Proposed algorithm for ship detection.

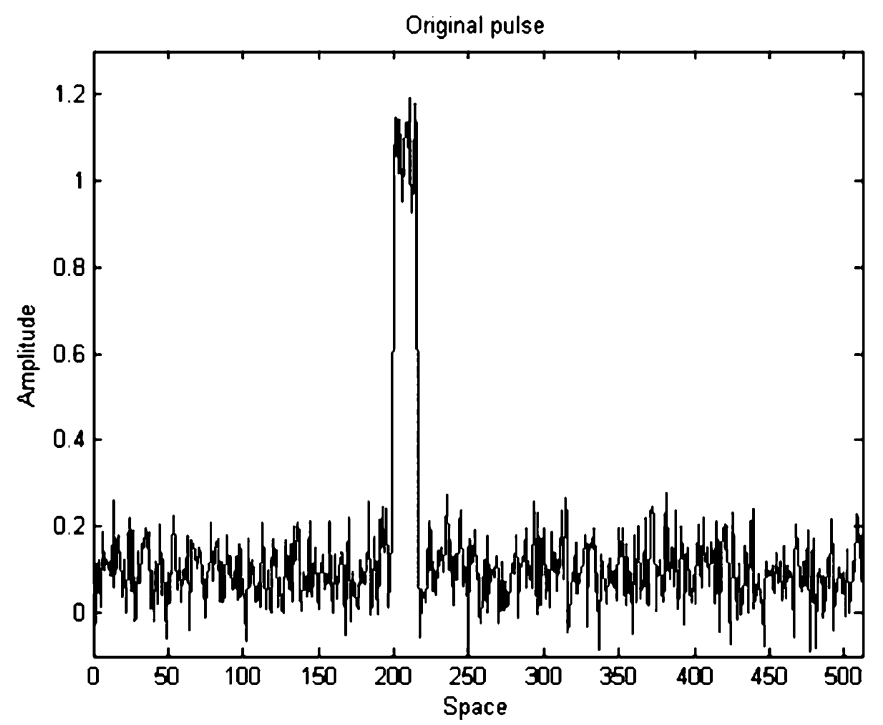

Fig. 4. Simulated model of a vessel surrounded by sea clutter.

By studying and comparing the properties of speckle and those of ship signatures, it can be observed that a vessel behaves as a deterministic pattern. This fact explains why the presence of a ship is usually noticeable in the three different subbands obtained when applying the 2-D WT and this kind of discontinuity can even be transmitted over scales-mathematically, a vessel can be expressed as a short pulse, with frequencial components in each subband. On the contrary, orthogonality between the different wavelet subspaces ensures quasi decorrelation of the speckle noise, behaving as a random distributed variable, identically distributed within each scale.

These previous considerations lead to the proposed method which consists of spatially multiplying the four components resulting from each iteration and proceeding to the detection directly in the wavelet domain [4], [5] (Fig. 3). In order to justify this solution, the consideration of its effect on a simplified one-dimensional model (Fig. 4) is analyzed (3).

The vessel is represented by a short pulse, $p, L$ samples long, $p_{L}(m)=\sum_{i=1}^{L} \delta(m-i)$ whereas the sea clutter, $n$ with mean amplitude $\sigma$, follows a Rayleigh distribution

$$
x[m]=p_{L}[m]+\sigma n[m] .
$$

When applying the WT, the process is the same for each iteration and it is constituted by two steps. Firstly, original data pass through a filtering step and then they are downsampled. For
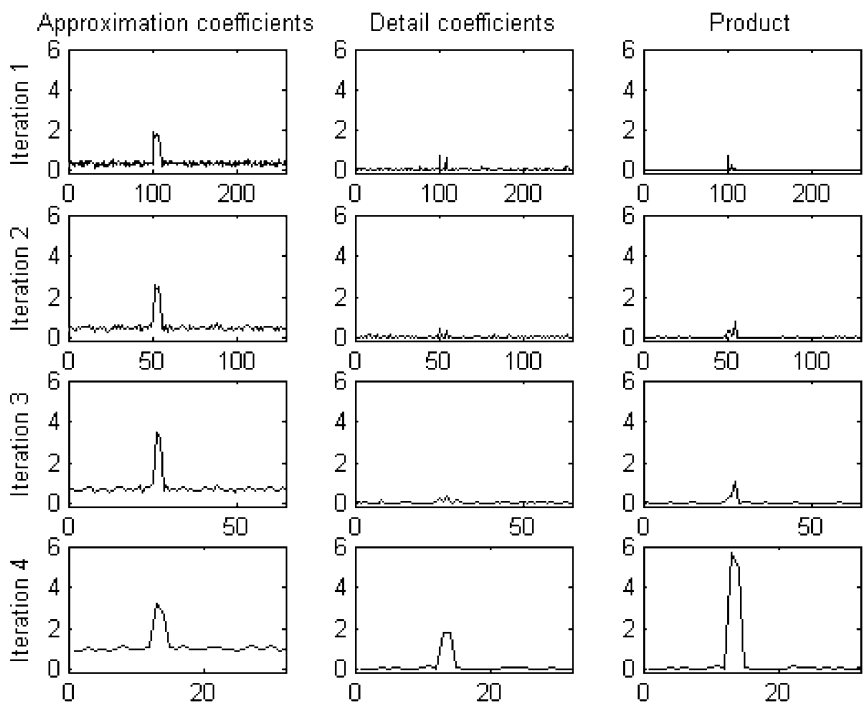

Fig. 5. Four iterations of the DWT with Haar coefficients applied to a pulse in a noisy background and result of the proposed algorithm.

a Haar wavelet [3], the expressions of the impulsional response for the low-pass and bandpass filters are

$$
h_{L}[m]=\frac{\left[\begin{array}{llll}
0 & 1 & 1 & 0
\end{array}\right]}{\sqrt{2}} \quad h_{H}[m]=\frac{\left[\begin{array}{llll}
0 & -1 & 1 & 0
\end{array}\right]}{\sqrt{2}} .
$$

The filtered signals $y_{L}$ and $y_{H}$ can be expressed as

$$
\begin{aligned}
& y_{L}[m]=\left(p_{L}[m]+\sigma n[m]\right) *\left(\frac{\delta[m-1]+\delta[m-2]}{\sqrt{2}}\right) \\
& y_{H}[m]=\left(p_{L}[m]+\sigma n[m]\right) *\left(\frac{-\delta[m-1]+\delta[m-2]}{\sqrt{2}}\right) .
\end{aligned}
$$

Defining $n_{L}$ and $n_{H}$ as low-pass and bandpass filtered noise components, respectively

$$
\begin{aligned}
& n_{L}=\sigma n[m] *\left(\frac{\delta[m-1]+\delta[m-2]}{\sqrt{2}}\right) \\
& n_{H}=\sigma n[m] *\left(\frac{-\delta[m-1]+\delta[m-2]}{\sqrt{2}}\right) .
\end{aligned}
$$

From (8), it can be deduced

$$
\begin{aligned}
y_{L}[m]= & \frac{1}{\sqrt{2}}\left(p_{L}[m-1]+p_{L}[m-2]\right)+n_{L} \\
= & \frac{1}{\sqrt{2}}(\delta[m-1]+\delta[m-(L+1)] \\
& \left.+2 p_{L-1}[m-2]\right)+n_{L} \\
y_{H}[m]= & \frac{1}{\sqrt{2}}\left(-p_{L}[m-1]+p_{L}[m-2]\right)+n_{H} \\
= & \frac{1}{\sqrt{2}}(-\delta[m-1]+\delta[m-(L+1)])+n_{H} .
\end{aligned}
$$

On the one hand, it can be seen from the equations in (10) that as the pulse in the approximation subband, $y_{L}$ becomes shorter with the increase of the number of iterations, so does the distance between the frontiers of the contour of the ship, coming to a point where they coincide and sum up their amplitudes as illustrated in Fig. 5. Pixels constituting a ship are intimately related, as they belong to the same structure, with proper intensity 


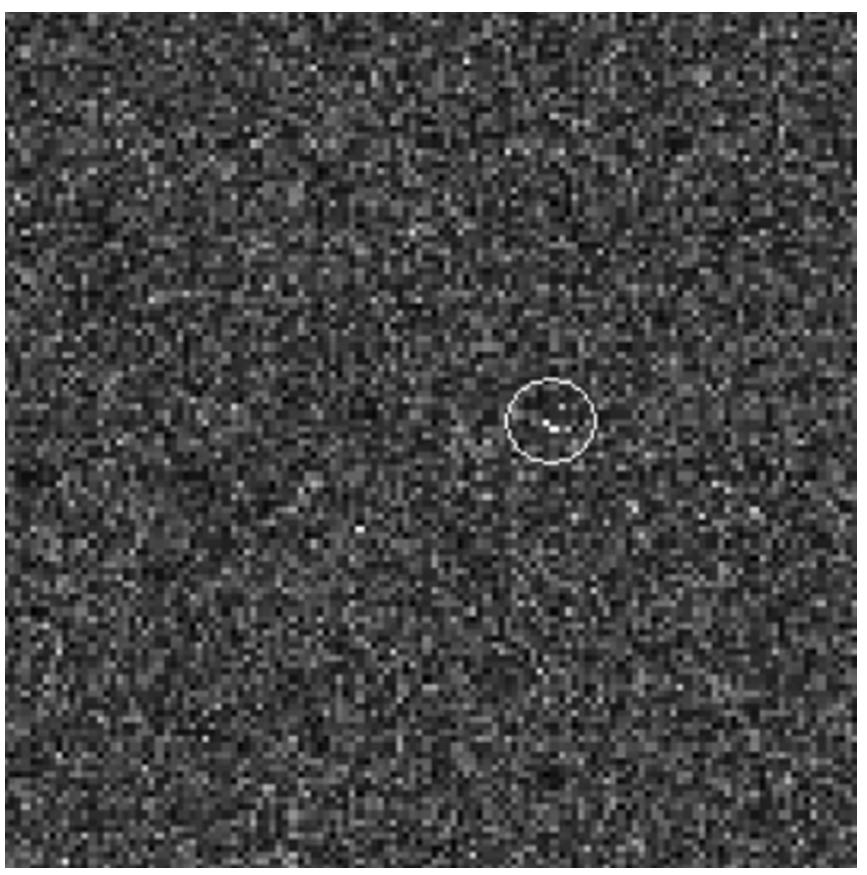

Fig. 6. Simulated image $(128 \times 128$ pixels) presenting a very small ship (two pixels) with low intensity (four times the mean of the image).

and statistical characteristics, so that the product operation after the application of the WT will stand out these deep relations. On the contrary, as it has been mentioned before, pixels constituting the background noise are randomly distributed. As the WT tend to be sparse, noise power is reduced due to the projection. Moreover, the correlation applied will produce much reduced values, attending to the poor probability of coincidence of related coefficients.

\section{EXPERIMENTAL RESULTS}

The proposed algorithm has been tested over a set of real Radarsat SAR images (acquired in ScanSAR mode, with a resolution of $50 \mathrm{~m}$ ) as well as over a simulated image, representing awkward situations for detection in which conventional algorithms based on the application of a threshold may fail. The position of vessels in real images was known through their reported VMS positions. In order to quantify the difficulty of performing a correct detection, a constrast parameter, called in the following the significance, is defined as

Significance $=\frac{\text { peak_of_the_target }- \text { background_mean }}{\text { background_standard_deviation }}$.

First, the proposed algorithm is tested on a simulated image presenting a small vessel with low contrast as shown in Fig. 6. After the application of the discrete wavelat transform (DWT) with Haar coefficients, four bands are obtained represented in Fig. 7. The final step consists on spatially multiplying these four matrices. As a result, the presence of the target is now clearly noticeable in Fig. 8. Another interesting aspect is the enlargement of the contrast, which may facilitate the decision rules.

The algorithm is then tested over real Radarsat and ENVISAT images in which the presence of the ships is known through
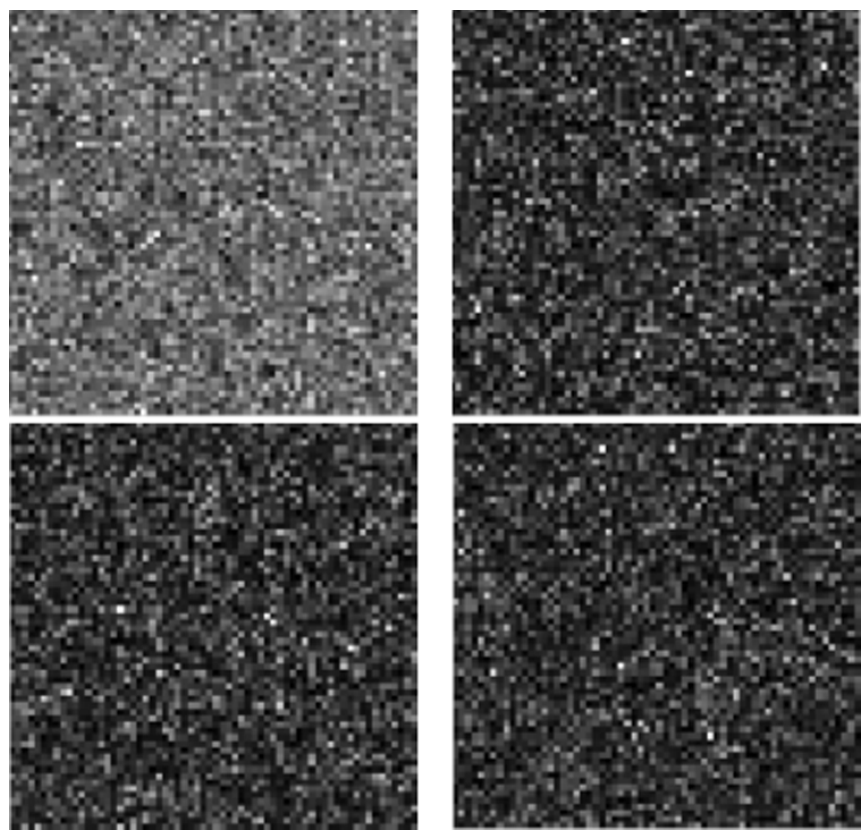

Fig. 7. Result of the application of the DWT to the simulated image presented in Fig. 6. The target is visible in none of the subbands.

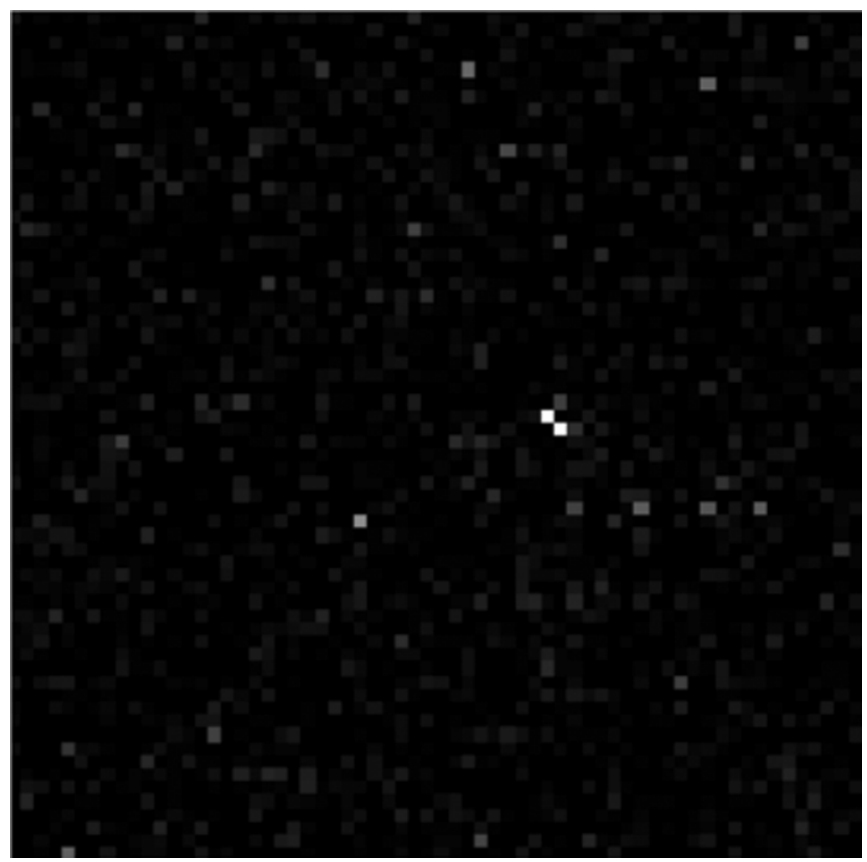

Fig. 8. Result of the application of the spatial correlation to the four components presented in Fig. 7. The presence of the ship is enhanced, whereas background noise is reduced.

reported VMS positions synchronized with data acquisition. It may be interesting to observe in Figs. 9-11 that the oceanic nonstationarity effects are reduced.

The enlargement of the dynamic range achieved by the proposed algorithm may be extremely useful for the subsequent decision step, expressed by the problematic and always debatable choice of a threshold. This critical issue, which probably constitutes one of the major drawbacks of automatic detection algorithms, is drastically simplified by the multiscale technique addressed in this paper. In order to provide a brief and graphical 

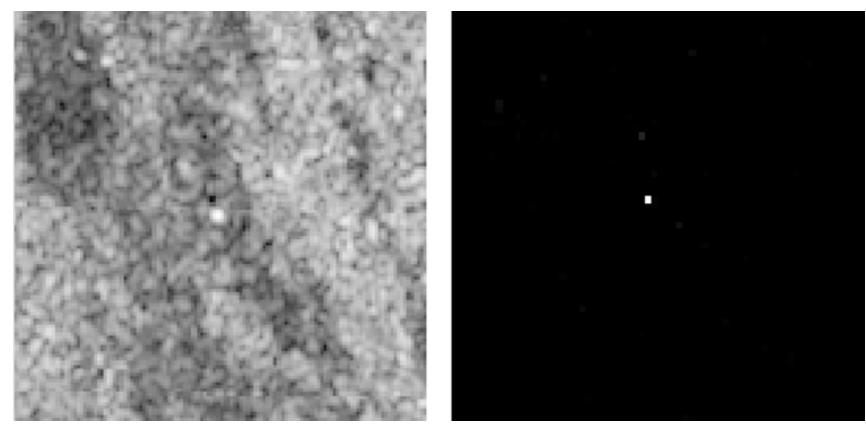

Fig. 9. (Left) Real Radarsat image $(128 \times 128$ pixels $)$ presenting a ship in a noisy background (Significance $=3.21$ ). (Right) Result of the application of the algorithm (Significance $=451.3$ ).
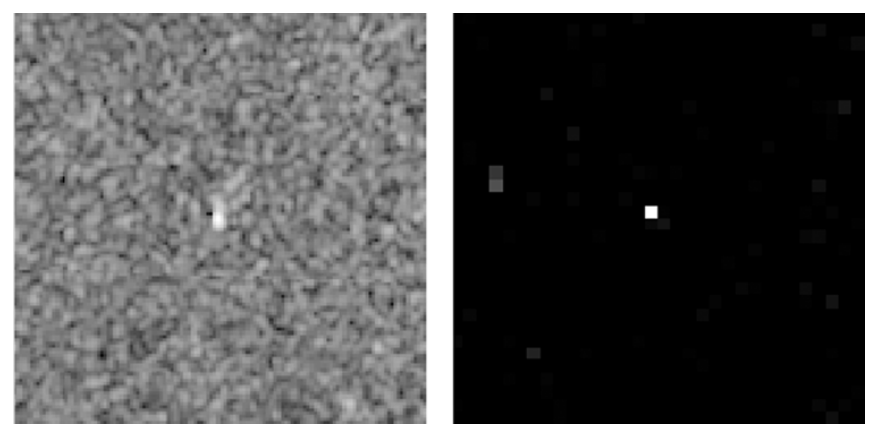

Fig. 10. (Left) Real Radarsat image $(128 \times 128$ pixels $)$ presenting a ship in a noisy background (Significance $=5.6$ ). (Right) Result of the application of the algorithm (Significance $=105$ ).

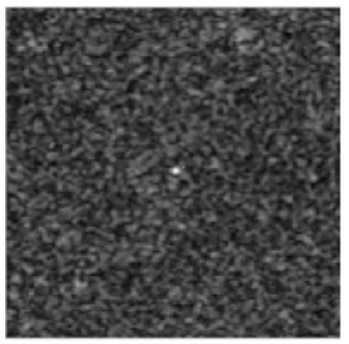

Significance $=9.1$

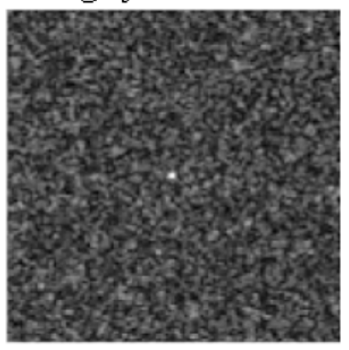

Significance $=8.4$

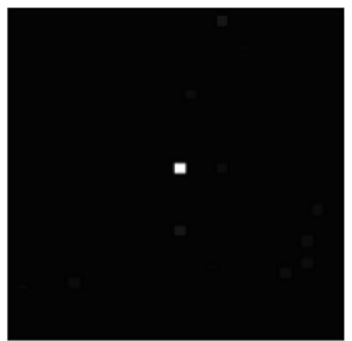

Significance $=154$

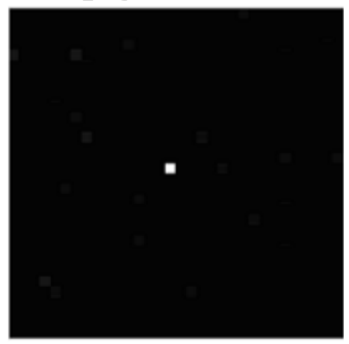

Significance $=1 / 3.4$
Fig. 11. (Left) Real ENVISAT images $(128 \times 128$ pixels). (Right) Result of the application of the algorithm.

illustration of this point, some cases showing a direct application of a threshold in the original image are presented in Fig. 12.

Threshold $T$ is most often calculated as [6]

$$
T=\mu+c \sigma
$$

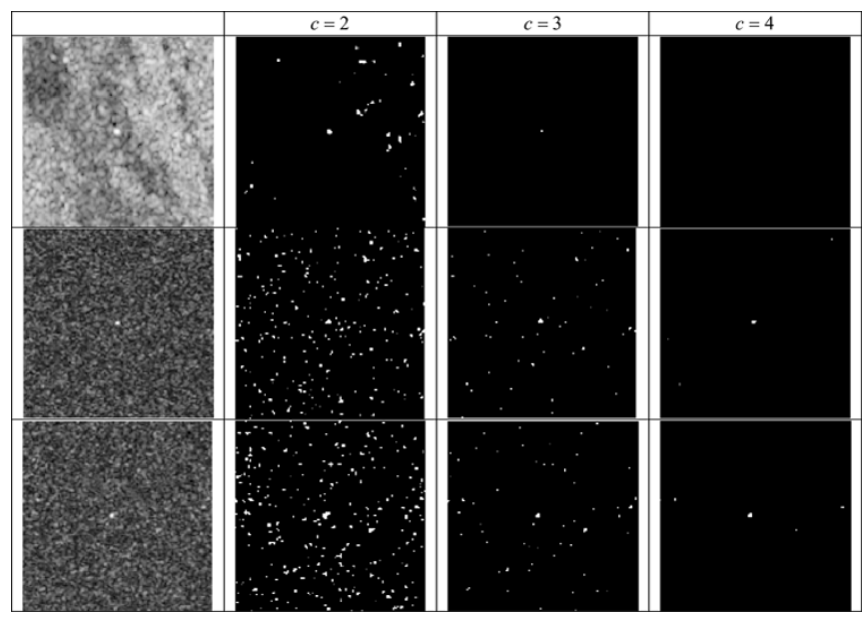

Fig. 12. Result of the application of different thresholds on three SAR images.

where $\mu$ stands for the mean of the image, whereas $\sigma$ is its standard deviation and $c$ is a parameter empirically adjusted. As it can be observed in Fig. 12, a threshold automatically assigned suitable in any case can not be considered. On the contrary, after the correlation of the subbands of the DWT, an easy adjustable threshold could be fixed, suitable in all the images tested, which provided a correct detection with no false alarms.

\section{CONCLUSION}

This paper presents a new algorithm for vessel detection based on the wavelet theory. This approach reaches a robust detection, taking advantage of the difference of statistical behavior of the vessels and the sea. More specifically, as a consequence of the spatial correlation of the subbands obtained after the application of the discrete wavelet transform, background noise is drastically reduced, whereas the presence of the ships, behaving as structured patterns, is greatly enhanced.

\section{ACKNOWLEDGMENT}

The authors would like to thank the responsibles of DECLIMS project for providing access to the database of SAR images with groundtruth and to $\mathrm{H}$. Greidanus for his helpful comments.

\section{REFERENCES}

[1] D. R. Montgomery, W. Pichel, and P. Clemente-Calon, "The use of satellite-based SAR in support of fisheries enforcement applications," in Proc. IGARSS, vol. 3, Jul. 6-10, 1998, pp. 1388-1390.

[2] H. Greidanus et al., "Benchmarking operational SAR ship detection," in Proc. IGARSS, vol. 6, 2004, pp. 4215-4218.

[3] S. Mallat, A Wavelet Tour of Signal Processing. Orlando, FL: Academic, 1999.

[4] M. Tello, C. Lopez-Martinez, and J. Mallorqui, "Ship detection in SAR imagery based on the wavelet transform," presented at the EUSAR 2004 Conf., 2004.

[5] M. Tello, C. Lopez-Martinez, J. Mallorqui, and A. Aguasca, "Use of the multiresolution capability of wavelets for ship detection in SAR imagery," in Proc. IGARSS, vol. 6, 2004, pp. 4247-4250.

[6] N. Kourti, G. Schwartz, and I. Shepherd, "Integrating active and passive satellite-based technologies to improve European fisheries monitoring and control," in Proc. IGARSS, vol. 4, 2002, pp. 2126-2128. 